Jahrbuch Schweiz - Dritte Welt 1995

\title{
Kohärenz in der schweizerischen Aussenpolitik - das Beispiel Indonesien
}

\section{Margrit Meier}

\section{(2) OpenEdition}

1 Journals

Electronic version

URL: http://journals.openedition.org/sjep/1313

DOI: $10.4000 /$ sjep.1313

ISSN: 1663-9677

Publisher

Institut de hautes études internationales et du développement

Printed version

Date of publication: 1 février 1995

Number of pages: 197-198

ISSN: 1660-5926

\section{Electronic reference}

Margrit Meier, « Kohärenz in der schweizerischen Aussenpolitik - das Beispiel Indonesien »,

Schweizerisches Jahrbuch für Entwicklungspolitik [Online], 14 | 1995, Online erschienen am: 05 Mai

2013, abgerufen am 08 September 2020. URL : http://journals.openedition.org/sjep/1313 ; DOI :

https://doi.org/10.4000/sjep.1313 


\title{
Kohärenz in der schweizerischen Aussenpolitik - das Beispiel Indonesien
}

\author{
Margrit Meier, Schweizerischer Gewerkschaftsbund
}

\section{Widersprüche}

Die Beziehungen der Schweiz zu Indonesien treffen auf folgende Widersprüche im Land: Indonesien verletzt in krasser Weise Menschenrechte und Prinzipien der Demokratie. Die gewerkschaftlichen Rechte sind nicht gewährleistet; Gewerkschaftsfunktionäre, Aktivistinnen und Aktivisten werden verhaftet. Es sind mindestens zwanzig Fälle von Verhaftungen bekannt von Mitgliedern der unabhängigen indonesischen Gewerkschaft für den Wohlstand der Arbeiter, SBSI (Serikat Buruh Sejahtera Indonesia). Im Vergleich zu anderen Asean-Ländern sind die allgemeine Schulbildung, das Gesundheitswesen und die Sozialdienste unterentwickelt. Grosse Widersprüche sind vorhanden zwischen dem Wachstum der Wirtschaft und der Bevölkerung einerseits und der Umweltentwicklung andererseits; sie stehen in krassem Gegensatz zueinander. Was die Entwicklungsprojekte anbelangt, so tragen diese wenig zur Verbesserung der Lage der Frauen in Indonesien bei.

Wie sind die Widersprüche aufzulösen?

Wenn sich Handelsströme nicht im Einklang entwickeln mit menschenrechtlichen, demokratischen und sozialen Prinzipien, so muss es Sache der internationalen Staatengemeinschaft werden, für Abhilfe zu sorgen. Multilaterale Massnahmen drängen sich in folgenden zwei Bereichen auf:

- Die Verknüpfung der Handelsregeln von GATTMTO (Meistbegünstigung) mit den sozialrechtlichen Mindeststandards der Internationalen Arbeitsorganisation (IAO), namentlich das Recht auf Vereinigungsfreiheit und auf Lohnverhandlungen, das Verbot von Kinderarbeit, von Zwangsarbeit und von Diskriminierungen.

- Die internationale Staatengemeinschaft soll über die Weltbank und die Asiatische Entwicklungsbank Einfluss nehmen auf die Ausgestaltung der Wirtschafts- und Sozialpolitik Indonesiens.

Die Schweiz kann bilaterale Einflussnahme geltend machen in ihren diplomatischen Kontakten, aber auch über das handelspolitische Instrumentarium. Nicht in Frage kommt der Abzug von Entwicklungshilfe, soweit diese ärmeren Bevölkerungsschichten zugute kommt. 


\section{Institutionelle Massnahmen}

Innerhalb der Bundesverwaltung sollten menschenrechtliche, sozial- und entwicklungspolitische Aspekte bei der Fixierung der schweizerischen Haltung bezüglich GATTMTO, IAO, Weltbank und IWF, OECD, Exportrisikogarantie vermehrt berücksichtigt werden. Diesbezügliche Lernprozesse setzen einen intensiven und permanenten Dialog unter den beteiligten Ämtern sowie mit der Schweizerischen Nationalbank voraus. Auf internationalem Parkett sei auf die Rolle des Internationalen Bundes Freier Gewerkschaften (IBFG) in Brüssel verwiesen, der die Situation bezüglich Menschenrechte und Gewerkschaftsrechte weltweit verfolgt. Der Schweizerische Gewerkschaftsbund kann sich in politischen Grundsatzfragen in der Regel den Empfehlungen des IBFG anschliessen. 